\title{
The Fungal Gene Avr9 and the Oomycete Gene inf1 Confer Avirulence to Potato Virus X on Tobacco
}

\author{
Sophien Kamoun, Guy Honée, Rob Weide, Richard Laugé, Miriam Kooman-Gersmann, \\ Koen de Groot, Francine Govers, and Pierre J. G. M. de Wit \\ Laboratory of Phytopathology and Graduate School Experimental Plant Sciences, Wageningen Agricultural \\ University, P.O. Box 8025, 6700 EE Wageningen, The Netherlands \\ Accepted 22 January 1999.
}

\begin{abstract}
The AVR9 peptide of the fungal pathogen Cladosporium fulvum and the INF1 protein of the oomycete pathogen Phytophthora infestans elicit the hypersensitive response (HR) on Cf9 tomato or $C f-9$ transgenic tobacco and on all cultivars of tobacco, respectively. Expression of either the functional $A v r 9$ or infl genes from engineered potato virus X (PVX) genomes resulted in localized HR lesions on tobacco plants responsive to the elicitors and inhibited spread of the recombinant virus. In contrast, PVX derivatives producing mutant forms of AVR9 and INF1 with reduced elicitor activity caused systemic necrotic and/or mosaic symptoms, and were unable to inhibit PVX spread. These results demonstrate that $\mathrm{HR}$ is a highly versatile defense mechanism active against unrelated pathogens irrespective of the $\mathrm{HR}$-inducing agent, and that resistance to recombinant PVX in tobacco is correlated with the strength of the transgene-encoded elicitor.
\end{abstract}

A central paradigm in the study of plant-microbe interactions is that specificity resides in recognition events but not in the expression of the hypersensitive response (HR) and other defense responses (Staskawicz et al. 1995). Despite a body of circumstantial evidence, this hypothesis has not been demonstrated directly through the expression of an avirulence gene in a totally unrelated pathogen. Here we used potato virus $\mathrm{X}$ (PVX), a virus pathogenic on many solanaceous plants, as a gene expression vector to test whether the avirulence genes Avr9 and infl from the fungal pathogen Cladosporium fulvum and the oomycete pathogen Phytophthora infestans, respectively, could confer avirulence to PVX on tobacco. The Avr9

Corresponding author: Pierre J. G. M. de Wit; Laboratory of Phytopathology, Wageningen Agricultural University, P.O. Box 8025, 6700 EE Wageningen, The Netherlands; Telephone: +31 317-483130; Fax: +31 317-483412; E-mail: Pierre.deWit@ medew.fyto.wau.nl

Present address of S. Kamoun: Department of Plant Pathology, The Ohio State University, Ohio Agricultural Research and Development Center, 1680 Madison Ave., Wooster, OH 44691, USA; E-mail: Kamoun.1@osu.edu.

Present address of M. Kooman-Gersmann: Novartis Seeds BV, P.O. Box 26, 1600 AA Enkhuizen, The Netherlands.

S. Kamoun and G. Honée contributed equally to this paper. gene encodes the race-specific AVR9 elicitor peptide (van Kan et al. 1991; van den Ackerveken et al. 1992), and is present in all $C$. fulvum races that are avirulent on Cf9 tomato genotypes. Upon injection, AVR9 induces HR in leaves of Cf9 tomato genotypes. The infl elicitin gene of $P$. infestans encodes the species-specific elicitor INF1 (Kamoun et al. 1997). Injection of INF1 into tobacco also results in HR (Kamoun et al. 1997). Engineered $P$. infestans strains deficient in the production of INF1 induce disease lesions when inoculated onto Nicotiana benthamiana, suggesting that INF1 elicitin is a species-specific avirulence factor (Kamoun et al. 1998). We examined whether the Avr9 and infl genes could confer avirulence to PVX on tobacco (Nicotiana tabacum), and whether mutant alleles of the genes encoding less active elicitors would be less potent in inhibiting PVX spread.

The PVX expression system allows transient expression of heterologous genes in solanaceous plants, as has been shown previously for the expression of the Avr9 gene of C. fulvum in Cf9 tomato genotypes (Hammond-Kosack et al. 1995; Kooman-Gersmann et al. 1997). $C f-9$ transgenic tobacco responds with HR to AVR9 injection (Hammond-Kosack et al. 1998). To determine the response of $C f-9$ transgenic tobacco to PVX::Avr9, we used tobacco (cv. Petite Havana SR1) transformed with a cosmid carrying the $C f-9$ gene. Ten plants originating from two independent transgenic Cf9 lines, 6A3 and 17A3, were inoculated with PVX::Avr9. Three days after inoculation, small necrotic lesions were visible on the inoculated leaves that did not increase in size (Fig. 1A) for the following 3 weeks. In addition, no symptoms were observed on the upper (systemic) leaves of these plants (Fig. 1B). Tobacco plants without the $C f-9$ transgene were readily infected by PVX::Avr9 and showed systemic mosaic symptoms typical of PVX infection (Table 1).

To test the effect of other protein elicitors on PVX infection of tobacco, we constructed a PVX::infl derivative containing a chimeric gene encoding the mature 98-amino-acid INF1 elicitin of $P$. infestans (Kamoun et al. 1997, 1998) fused to the $P R-1 a$ signal peptide for extracellular targeting in a manner similar to that described for PVX::Avr9 (Kooman-Gersmann et al. 1997). A fragment containing the $P R-1 a$ sequence was amplified by polymerase chain reaction (PCR) from PVX::Avr9 with primers OX10 (5'-CAATCACAGTGT TGGCTTGC-3') and PR1-INF1A (5'-GTGGTGCACGTGG 
TGGCACGGCAAGAGTGGGATATTAC-3') and a fragment containing the infl sequence was amplified from the infl cDNA clone pFB7 (Kamoun et al. 1997) with primers PR1INF1B (5'-CTTGCCGTGCCACCACGTGCACCACCTCG$\left.3^{\prime}\right)$ and SK-R2 (5'-GCGAAGCTTATCGATCATAGCGACG CACACGTAGA-3'). One recombinant plasmid, pPVX::infl, was confirmed by DNA sequencing to have a PR1a-infl fusion inserted in sense orientation between the duplicated PVX coat protein promoters. Infectious PVX::infl virions were prepared as described by Kooman-Gersmann et al. (1997) and inoculated onto leaves of tobacco lines (Table 1). Similarly to PVX::Avr9 on Cf9 tobacco, localized necrotic lesions were visible on tobacco leaves 3 days after inoculation (Fig. 1C) and no systemic symptoms developed later during infection (Fig. 1D).

To determine whether PVX::Avr9 and PVX::inf1 remained localized to the inoculated leaves, systemic leaves from Cf9 tobacco plants inoculated with PVX::Avr9 and tobacco plants inoculated with PVX::infl were harvested, and leaf homogenates were prepared as described by Kooman-Gersmann et al. (1997). The homogenates were inoculated onto MM-Cf9 tomato for PVX::Avr9 and onto tobacco for PVX::inf1. No necrotic or mosaic symptoms were observed on either plant (data not shown), suggesting that no viable virions had spread systemically in the initially inoculated plants.

The observed local lesions and the absence of systemic spread of PVX derivatives encoding the two active elicitors on tobacco are reminiscent of HR-mediated resistances induced in plants against viruses, such as the resistance of $N$ genecontaining tobacco to TMV (Whitham et al. 1994). Apparently, the Avr9 gene expressed in PVX can confer avirulence to PVX on tobacco plants expressing the $C f-9$ gene and the infl gene expressed in PVX can confer avirulence to PVX on tobacco plants.

If resistance to PVX is correlated with HR, it can be hypothesized that PVX derivatives expressing mutants of Avr9 and infl encoding less active elicitors would allow larger lesions and systemic spread of PVX. A number of PVX::Avr9 derivatives expressing Avr9 mutants with altered elicitor activity have been described by Kooman-Gersmann et al. (1997). To test the relationship between the strength of elicitor activity and the ability to confer avirulence to PVX on Cf9 tobacco, we inoculated five PVX::Avr9 mutants onto Cf9 tobacco (Table 1). Two mutants, PVX::Avr9F10S and PVX::Avr9L24S, with lowest elicitor activity (KoomanGersmann et al. 1997), showed altered symptoms upon inoculation onto Cf9 tobacco when compared with PVX::Avr9 (Table 1). On the inoculated leaves, both mutants produced necrotic spots similar to those produced by PVX::Avr9 (Fig. 1A,E). However, in contrast to PVX::Avr9, symptoms extended to the systemic leaves. PVX::Avr9F10S produced systemic necrosis similar to that obtained on Cf9 tomato with PVX::Avr9 (data not shown), while PVX::Avr9L24S, which induces no necrosis on $\mathrm{Cf} 9$ tomato, produced systemic mosaic symptoms accompanied by minute necrotic spots (Fig. 1F). Apparently, the low elicitor activity of these two mutant AVR9 peptides was not sufficient to confer avirulence to PVX, and systemic spread of the recombinant virions occurred throughout the plant. Three mutants, PVX::Avr9R08K, PVX::Avr9H22L and PVX::Avr9N03A, which still have significant elicitor activity on MM-Cf9 tomato (Kooman-Gersmann et al. 1997), produced local necrotic lesions on Cf9 tobacco similar to those produced by PVX::Avr9 (Table 1).

Elicitins, such as INF1, are globular proteins with three highly conserved disulfide bridges (Boissy et al. 1996). Exchange of one of the cysteine residues in INF1 results in strong reduction of elicitor activity (Kamoun et al. 1997). In order to test whether a PVX::inf1 mutant with reduced elicitor activity can or cannot confer avirulence to PVX on tobacco, we constructed PVX::inf1C95S, a mutant derivative in which cysteine residue 95 has been exchanged by serine. Recombinant PVX::inflC95S virions were obtained as described above for PVX::infl, except that the PCR-mediated overlap extension involved the mutagenic primer SK-R2S (5'GCGAAGCTTATCGATCATAGCGACGCACTCGTAGA-3') instead of SK-R2, and were inoculated onto tobacco lines (Table 1). In contrast to the small local necrotic lesions ob-

Table 1. Summary of symptoms observed on Cf9 tobacco and wild-type tobacco after inoculation with potato virus X (PVX) and PVX expressing Avr9 or infl wild-type and mutant genes

\begin{tabular}{|c|c|c|c|c|c|}
\hline \multirow{3}{*}{$\begin{array}{l}\text { Insert gene in } \\
\text { PVX }^{\mathbf{a}}\end{array}$} & \multirow{3}{*}{$\begin{array}{r}\text { Elicitor } \\
\text { activity }^{\text {b }}\end{array}$} & \multicolumn{4}{|c|}{ PVX inoculation } \\
\hline & & \multicolumn{2}{|c|}{ Symptoms on Cf9 tobacco ${ }^{c}$} & \multicolumn{2}{|c|}{ Symptoms on tobacco $^{d}$} \\
\hline & & Inoculated leaves & Systemic leaves & Inoculated leaves & Systemic leaves \\
\hline None & . & No visible symptoms & Mosaic symptoms & No visible symptoms & Mosaic symptoms \\
\hline Avr9 (wild type) & ++++ & Necrotic lesions & No visible symptoms & No visible symptoms & Mosaic symptoms \\
\hline Avr9N03A & ++ & Necrotic lesions & No visible symptoms & ND & ND \\
\hline Avr9R08K & +++++ & Necrotic lesions & No visible symptoms & ND & ND \\
\hline Avr9F10S & + & Necrotic lesions & Necrosis and chlorosis & ND & ND \\
\hline Avr9H22L & +++ & Necrotic lesions & No visible symptoms & ND & ND \\
\hline Avr9L24S & - & Necrotic lesions & Mosaic and small necrotic lesions & ND & ND \\
\hline infl (wild type) & ++++ & Necrotic lesions & No visible symptoms & Necrotic lesions & No visible symptoms \\
\hline inflC95S & + & Large necrotic lesions & Necrosis & Large necrotic lesions & Necrosis \\
\hline
\end{tabular}

${ }^{a}$ Gene constructs inserted into the ClaI site of the PVX vector pTX(GC3A (Baulcombe et al. 1993). Avr 9 corresponds to the PR-1a signal sequence fused to the sequence corresponding to the mature AVR9 peptide (Hammond-Kosack et al. 1995). infl corresponds to the PR-1a signal sequence fused to the sequence corresponding to the mature INF1 protein (Kamoun et al. 1997).

${ }^{\mathrm{b}}$ Relative elicitor or necrosis-inducing activity of AVR9 mutants based on injection assays of Cf9 tomato described by Kooman-Gersman et al. (1997). Relative elicitor activity of INF1 and INF1C95S based on infiltration experiments of tobacco described by Kamoun et al. (1997) and experiments described in this study. $-=$ not active; $+++++=$ most active .

${ }^{c}$ Nicotiana tabacum cv. Petite Havana SR1 transformed with Cf-9 (line 1096).

${ }^{\mathrm{d}}$ N. tabacum cv. Petite Havana SR1. ND = not determined. 
served with PVX::infl (Fig. 1C), large necrotic lesions were observed on leaves inoculated with PVX::inflC95S 3 to 6 days after inoculation (Fig. 1G), which later extended to the stem and systemic leaves (Fig. 1H). These results indicate that the inflC95S gene is unable to confer avirulence to PVX in tobacco and allows spread of PVX, resulting in systemic necrosis.

In this study, we have demonstrated that the fungal elicitor AVR9 and the oomycete elicitor INF1 confer specific avirulence to PVX on tobacco. Our results support the view that
HR-based disease resistance is a conserved mechanism in plants that is effective against unrelated pathogens. Clearly, $\mathrm{HR}$ and other defense responses triggered by particular elicitor molecules can be equally effective against fungal, oomycete, and viral plant pathogens.

Our results show that gene-for-gene interactions involving fungal pathogens and plants can be transferred to a different pathosystem, i.e., from $C$. fulvum-tomato or P. infestanstobacco to PVX-tobacco. As predicted by the gene-for-gene model for the Avr9/Cf-9 gene pair (van den Ackerveken 1992;
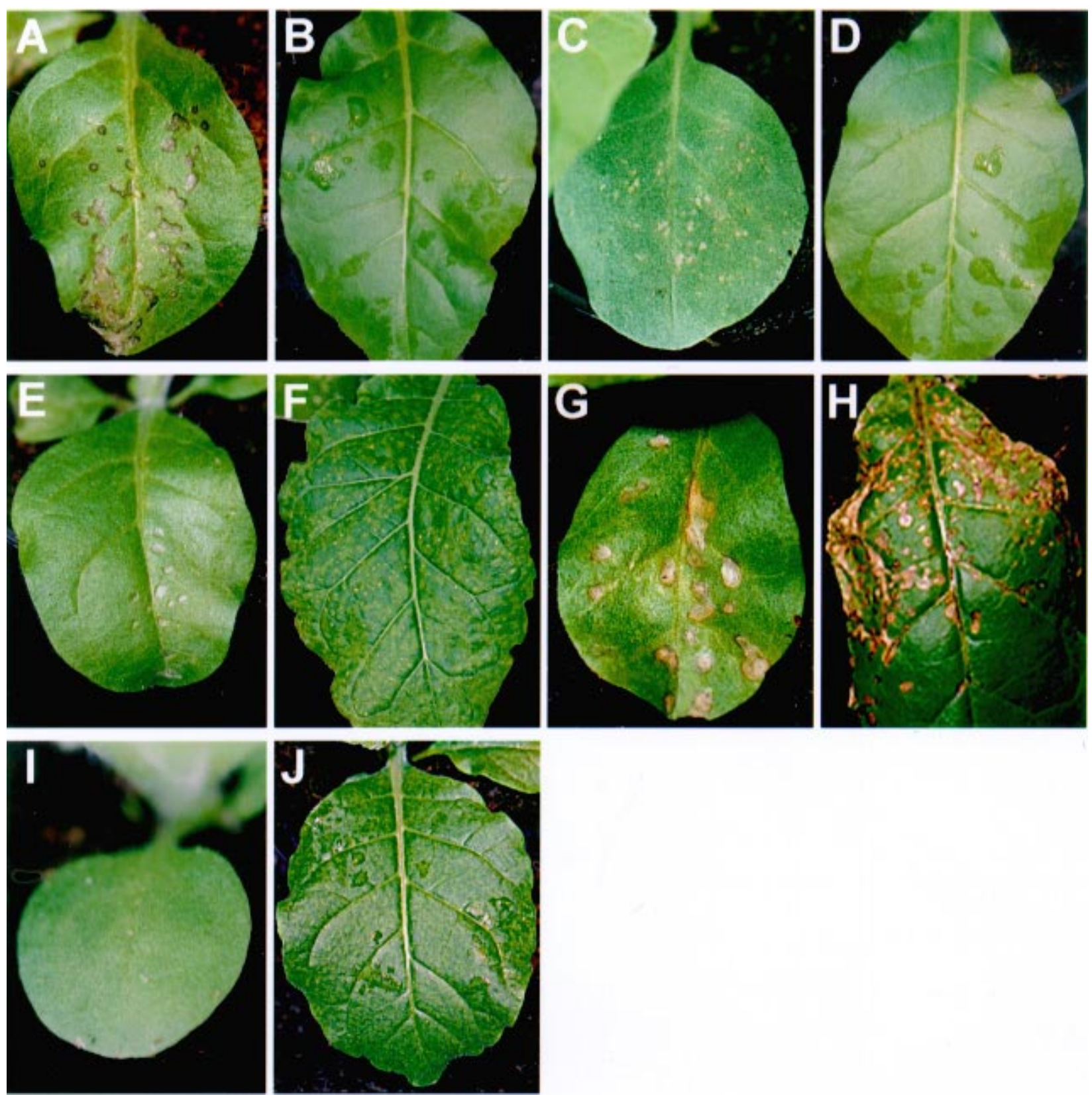

Fig. 1. Symptoms observed on $\mathrm{Cf} 9$ tobacco plants after inoculation with potato virus $\mathrm{X}$ derivatives expressing Avr 9 or infl wild-type and mutant genes. Inoculated leaves from (A, E, and I) Nicotiana tabacum cv. Petite Havana SR1 line 1096 (containing a cosmid with a genomic copy of $C f-9)$ and $(\mathbf{C}$ and G) $N$. tabacum cv. Petite Havana SR1 were photographed 6 days after inoculation. Representative systemic leaves of the inoculated (B, F, and J) Petite Havana SR1 1096 and (D and H) Petite Havana SR1 plants were photographed 21 days after inoculation. A and B, PVX::Avr9. C and D, PVX::inf1. E and E, PVX::Avr9L24S. G and H, PVX::inflC95S. I and J, PVX wild type. A, C, E, and G, Leaves show local necrotic lesions. B, D, and I, Leaves show no visible symptoms. F, Leaf shows mosaic symptoms and small necrotic lesions. H, Leaf shows systemic necrosis. J, Leaf shows mosaic symptoms. 
de Wit 1992, 1997), the absence of either $A v r 9$ or $C f-9$ leads to mosaic disease symptoms and systemic spread of the virus (Table 1). Similarly, infl, an avirulence gene that confers avirulence to $P$. infestans in solanaceous plants at the species level (Kamoun et al. 1993, 1998), also confers avirulence to PVX on tobacco but not on tomato, a plant species not responding to INF1 (Table 1 and Fig. 1C,D; and data not shown).

Mutants of AVR9 and INF1 with reduced elicitor activity lost the ability to inhibit PVX spread in tobacco. These results strongly support the hypothesis that the effectiveness of the HR-mediated resistance against PVX derivatives in tobacco depends on the strength of the elicitor encoded by the recombinant virus.

The incompatible interaction between PVX::Avr9 and Cf9 tobacco noted in this study sharply contrasts with the previously examined interaction between PVX::Avr9 and Cf9 tomato genotypes (Hammond-Kosack et al. 1995; KoomanGersmann et al. 1997). On Cf9 tomato PVX::Avr9 does not remain localized to the site of infection but spreads and causes systemic HR. This discrepancy could result from different levels of sensitivity of these two plant species to the AVR9 peptide, as has been reported by Hammond-Kosack et al. (1998), which could originate from a more efficient $C f-9$ mediated defense signaling pathway in tobacco than in tomato. Alternatively, differences in leaf structure between the two host plants might have different implications for virus replication and spread, leading to localization in tobacco but not in tomato.

\section{ACKNOWLEDGMENTS}

We thank Jonathan Jones for providing SLJ 8146 containing the genomic $C f-9$ sequence on a $7.5-\mathrm{kb}$ Pst I fragment. We thank Matt Krause and Leona Horst for assistance with Figure 1, Tony van Kampen for sequencing the constructs, and Rick Lubbers for excellent assistance with greenhouse facilities. S. K., K. d. G., and M. K.-G. were supported by grants from the Netherlands Organization for Scientific Research, Technology Foundation (NWO-STW) and Council Earth and Life Sciences (NWO-ALW; projects 805-22-767 and 805-22755). G. H. was supported by the Ministry of Economic Affairs, the Ministry of Education, Culture and Science, the Ministry of Agriculture, Nature Management and Fishery in the framework of an industrial relevant research program of the Netherlands Association of Biotechnology Centers in the Netherlands (ABON). R. L. was supported by a grant from the European Community-Human Capital and Mobility Program (project ERBCHRXCT930244).

\section{LITERATURE CITED}

Baulcombe, D. C., Lloyd, J., Manoussopoulos, I. N., Roberts, I. M., and Harrison, B. D. 1993. Signal for potyvirus-dependent aphid transmis sion of potato acuba mosaic virus and the effect of its transfer to potato virus X. J. Gen. Virol. 74:1245-1253.

Boissy, G., de La Fortelle, E., Kahn, R., Huet, J.-C., Bricogne, G., Pernollet, J.-C., and Brunie, S. 1996. Crystal structure of a fungal elicitor secreted by Phytophthora cryptogea, a member of a novel class of plant necrotic proteins. Structure 4:1429-1439.

de Wit, P. J. G. M. 1992. Molecular characterization of gene-for-gene systems in plant-fungus interactions and the application of avirulence genes in control of plant pathogens. Annu. Rev. Phytopathol. 30:391-418.

de Wit, P. J. G. M. 1997. Pathogen avirulence and plant resistance: A key role for recognition. Trends Plant Sci. 2:452-458.

Hammond-Kosack, K. E., Staskawicz, B. J., Jones, J. D. G., and Baulcombe, D. C. 1995. Functional expression of a fungal avirulence gene from a modified potato virus X genome. Mol. Plant-Microbe Interact. 8:181-185.

Hammond-Kosack, K. E., Tang, S., Harrison, K., and Jones, J. D. G. 1998. The tomato $C f-9$ disease resistance gene functions in tobacco and potato to confer responsiveness to the fungal avirulence gene product Avr9. Plant Cell 10:1251-1266.

Kamoun, S., van West, P., de Jong, A. J., de Groot, K. E., Vleeshouwers, V. G. A. A., and Govers, F. 1997. A gene encoding a protein elicitor of Phytophthora infestans is down-regulated during infection of potato. Mol. Plant-Microbe Interact. 10:13-20.

Kamoun, S., van West, P., Vleeshouwers, V., de Groot, K., and Govers, F. 1998. Resistance of Nicotiana benthamiana to Phytophthora infestans is mediated by the recognition of the elicitor protein INF1. Plant Cell 10:1413-1425.

Kamoun, S., Young, M., Glascock, C. B., and Tyler, B. M. 1993. Extracellular protein elicitors from Phytophthora: Host-specificity and induction of resistance to fungal and bacterial phytopathogens. Mol. Plant-Microbe Interact. 6:15-25.

Kooman-Gersmann, M., Vogelsang, R., Hoogendijk, E. C. M., and de Wit, P. J. G. M. 1997. Assignment of amino acid residues of the AVR9 peptide of Cladosporium fulvum that determine elicitor activity. Mol. Plant-Microbe Interact. 10:821-829.

Staskawicz, B. J., Ausubel, F. M., Baker, B. J., Ellis, J. G., and Jones, J. D. G. 1995. Molecular genetics of plant disease resistance. Science 268:661-667.

van den Ackerveken, G. F. J. M., van Kan, J. A. L. and de Wit, P. J. G. M. 1992. Molecular analysis of the avirulence gene $a v r 9$ of the fungal tomato pathogen Cladosporium fulvum fully supports the gene-forgene hypothesis. Plant J. 2: 359-366.

van Kan, J. A. L., van den Ackerveken, G. F. J. M., and de Wit, P. J. G. M. 1991. Cloning and characterization of cDNA of avirulence gene avr 9 in tomato pathogen Cladosporium fulvum, causal agent of tomato leaf mold. Mol. Plant-Microbe Interact. 4: 52-59.

Whitham, S., Dinesh-Kumar, S. P., Choi, D., Hehl, R., Corr, C., and Baker, B. 1994. The product of the tobacco mosaic virus resistance gene $N$ : Similarity to Toll and the Interleukin-1 receptor. Cell 78: 1101-1115. 